\title{
Structure and Migration of 2D Vortices
}

\author{
Clément Surville $\mathrm{e}^{1,2, \mathrm{a}}$ and Pierre Barge $\mathrm{e}^{2, \mathrm{~b}}$ \\ ${ }^{1}$ Max Planck Institut für Astronomie, Königsthul 17, 69117 Heidelberg, Germany \\ ${ }^{2}$ Aix Marseille Université, CNRS, Laboratoire d'Astrophysique de Marseille, UMR 7326, 13388, Marseille, \\ France
}

\begin{abstract}
The evolution of 2D vortices is studied in the case of an adiabatic disk. A new vortex model is derived using a gaussian assumption and tested against numerical solutions. This model is compared with previous ones and used as an initial solution for an accurate second order numerical method. Three parameters are necessary to constrain the model: the radial extent of the vortex, its aspect ratio and the local Rossby number. We explore the space of the parameters and find that two different families of vortices can be distinguished: (i) large elongated vortices and (ii) small compact vortices. The shape of the vortices is nearly unchanged even after thousands of rotations. We find that $2 \mathrm{D}$ vortices migrate inward at a nearly constant rate that depends on the disk parameters and on the vortex aspect ratio. They appear as quasi-steady structures in Keplerian disks.
\end{abstract}

\section{Introduction}

Two outstanding issues in the evolution of protoplanetary disks are the growth of the dust grains and the transport of the angular momentum. A number of mechanisms lead to the formation of vortices ([2], [9], [8]) that can act as particles trap ([1], [6], [5]) and also contribute to the transport of angular momentum by the excitation of density waves. These vortices can speed up particles growth by locally increasing the dust to gas ratio; they could also participate to the accretion onto the star at a rate compatible with the observations [8]. Nevertheless the lifetime and the dynamical evolution of these vortices is still an open question. Theoretical and numerical studies already showed that 2D vortices can survive hundred of rotations, in fact in relation with the viscous time-scale of the disk and the numerical diffusion. For example, inside the dead-zone, the gas is nearly inviscid and vortices should survive on longer time scales than in other disk regions. In the first section of the paper we derive an approximate vortex solution of the Euler's equation that better fits the numerical solutions than previous incompressible models. Many simulations were performed to study the dynamical evolution of the vortices and to explore the importance of the various parameters. Two families of vortices have been distinguished following the compression of the ambient gas. Finally, in section 3, vortex migration is discussed as a function of the various parameters.

\footnotetext{
a e-mail: surville@mpia-hd.mpg.de

be-mail: barge@oamp.fr
} permits unrestricted use, distribution, and reproduction in any medium, provided the original work is properly cited. 


\section{Euler's equations and vortex model}

We use a standard model of protoplanetary disks in which surface density, temperature and consequently pressure decrease as power laws of the distance to the star $r$. We get: $\sigma_{0}(r) \propto\left(r / r_{0}\right)^{\beta_{\sigma}}$, $T_{0}(r) \propto\left(r / r_{0}\right)^{\beta_{T}}, P_{0}(r)=k_{B} T_{0}(r) \sigma_{0}(r)$ and assume that $\beta_{\sigma}=-3 / 2$ and $\beta_{T}=-1 / 2$. The density and temperature at the reference radius $r_{0}$ lead to a scale height $H_{0}\left(r_{0}\right)=\sqrt{\gamma P_{0}\left(r_{0}\right) / \sigma_{0}\left(r_{0}\right)} / \Omega_{0}\left(r_{0}\right)=$ $0.065 r_{0}$, where $\gamma=1.4$ is the polytropic index of the gas. The angular velocity $\Omega_{0}(r) \sim\left(r / r_{0}\right)^{\beta_{\Omega}}$ results from the equilibrium between the centrifugal force, the stellar potential and the pressure gradient:

$$
\Omega_{0}(r)=\frac{1}{r}\left(\frac{G M_{\star}}{r}+\frac{r}{\sigma_{0}(r)} \partial_{r} P_{0}(r)\right)^{1 / 2}
$$

The dynamical evolution of the gas is described using the compressible fluid equations: continuity, Euler's equation and an energy equation for an adiabatic disk; this last equation closes the system since pressure and density are related. We use a cylindrical system of coordinates centered on the star. At steady state density and pressure obey power laws $\left(\sigma_{0}(r)\right.$ and $\left.P_{0}(r)\right)$ and the gas is flowing at the velocity $\vec{V}_{0}=0 \vec{e}_{r}+r \Omega_{0}(r) \vec{e}_{\theta}$.

Then, in a frame of reference rotating at $\Omega_{0}\left(r_{0}\right)$, the system of equation reduces to:

$$
\begin{aligned}
& u \partial_{r} \sigma+\frac{v}{r} \partial_{\theta} \sigma+\sigma \vec{\nabla} \cdot \vec{v}=0 \\
& u \partial_{r} u+\frac{v}{r} \partial_{\theta} u=\frac{v^{2}}{r}+2 \Omega_{0}\left(r_{0}\right) v-\left(\Omega_{0}(r)^{2}-\Omega_{0}\left(r_{0}\right)^{2}\right) r-\frac{1}{\sigma} \partial_{r} P+\frac{1}{\sigma_{0}(r)} \partial_{r} P_{0}(r) \\
& u \partial_{r} v+\frac{v}{r} \partial_{\theta} v=-\frac{u v}{r}-2 \Omega_{0}\left(r_{0}\right) u-\frac{1}{\sigma r} \partial_{\theta} P
\end{aligned}
$$

where $u$ and $v$ are the radial and azimuthal components of the velocity, respectively, and where it is also assumed that $\partial_{t}+\Omega_{0}\left(r_{0}\right) \partial_{\theta} \equiv 0$. We are now looking for approximate solutions of the above equations that will be compared to numerical solutions obtained from an integration of the complete set of the fluid equations with a second order finite volume method.

\subsection{The vortex model}

Previous models [7] and [4] (thereafter GNG) start from the incompressible form of the above equations and use the shearing sheet approximation. This leads to a solution in elliptical streamlines with $u=\chi^{-1} \Omega_{v} r_{0}\left(\theta-\theta_{0}\right)$ and $v=-\chi \Omega_{v}\left(r-r_{0}\right)$, where $\Omega_{v}$ is the angular velocity inside the vortex and $\chi$ is the aspect ratio of the elliptical patch. Besides, GNG also derived a parabolic approximation of the pressure profile but the velocity field presents a discontinuity that is not suitable for numerical studies.

Here, we propose a more complete model based on an approximate solution of the above equations. To this end, we will set $v=v^{\prime}+V_{0}(r)-r \Omega_{0}\left(r_{0}\right)$ and define a local Rossby number $R_{0}=\omega /\left(2 \Omega_{0}\right)$, where $\omega$ is the vorticity with respect to the rotating frame. We obtain:

$$
\begin{aligned}
& v^{\prime} 2 \Omega_{0}=\partial_{r} \mathcal{H}+\frac{(\gamma-1) \beta_{\sigma}-\beta_{T}}{\gamma} \frac{\mathcal{H}}{r} \\
& u 2 \Omega_{0}=-\left(R_{0}+1+\beta_{\Omega} / 2\right)^{-1} \frac{1}{r} \partial_{\theta} \mathcal{H}
\end{aligned}
$$

where $\mathcal{H}$ is a pseudo-enthalpy that we assume to be gaussian in the radial and the azimuthal directions. It reads: 

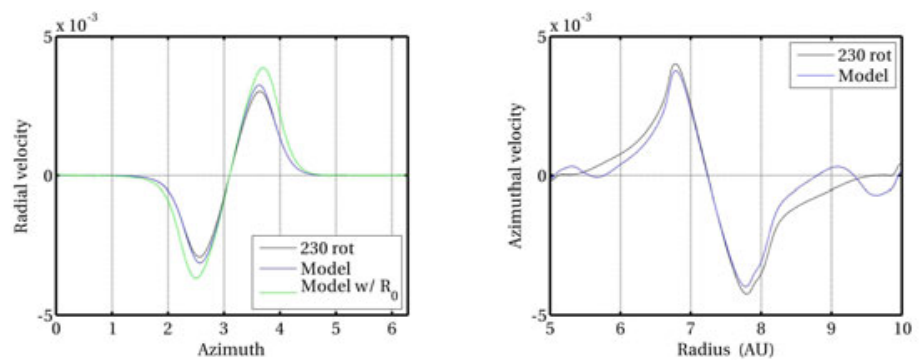

Figure 1. From left to right, the radial and the azimuthal components of the velocity, respectively. The numerical values are plotted in black; the values issued from the model are plotted in green or in blue following the Rossby number is defined as the minimum of the vorticity or as space varying function, respectively. Agreement between the black and the green curves is, at most, of the order of $10 \%$; agreement between the black and the blue curves is, at most, of the order of $5 \%$.

$$
\mathcal{H}=\mathcal{H}_{0} \exp \left[-\chi_{r}^{-2}\left[\left(\frac{r}{r_{0}}-1\right)^{2}+\chi_{\theta}^{-2}\left(\theta-\theta_{0}\right)^{2}\right]\right]
$$

where:

$$
\mathcal{H}_{0}=-2 R_{0}\left(\Omega\left(r_{0}\right) r_{0} \chi_{r}\right)^{2}\left[1+\left(\chi_{\theta}^{2}\left(R_{0}+1+\beta_{\Omega} / 2\right)\right)^{-1}\right]^{-1}
$$

Thus the vortex can be defined by three independent parameters: a non-dimensional radial extent $\chi_{r}=\Delta_{r} / r_{0}$, an aspect ratio $\chi_{\theta}$ and a Rossby number $R_{0}$. We have simulated more than 250 vortices with parameters: $0.01<\chi_{r}<0.15,2.5<\chi_{\theta}<18$ and $-0.23<R_{0}<-0.05$. The radial extent of vortices ranges from a tenth of the scale height to more than two scales height. These vortices appears as stable and quasi-steady structures. If the parameters are not appropriately chosen, the initial vortex gently relaxes to another solution, usually by a change of its aspect ratio. We found also that the aspect ratio approximately varies as $1 /\left|R_{0}\right|$. Figure 1 shows how well the velocity profile deduced from the models fits the numerical values. The fit is very good in the central region of the vortex but also in the outer regions where the vortex joins the background; this is in contrast with the previous models.

\subsection{Two vortex families}

The evolution of the vortices was followed numerically for $\sim 200$ rotations (up to 1000 rotations in some cases) and for a wide range of the possible parameters. From our sample two families of vortices can be distinguished as shown in figure 2 . The family of incompressible vortices corresponds to big vortices with large $\chi_{\theta}$ and $R_{0} \simeq-0.1$; they have closed streamlines and excite only very weak density waves. The family of compressible vortices corresponds to small vortices with small $\chi_{\theta}$ and $R_{0}<-0.15$; they have spiral streamlines and a density bump whose amplitude is similar to the amplitude of the density waves. From our simulations we found that the vortex parameters change by less than $10 \%$ during their evolution, even if the vortex is moving radially in the disk.

\section{Vortex migration}

Compressible vortices can excite spiral density waves that transport angular momentum outward. So, they can loss angular momentum and lead to an inward migration [10]. Measuring a mean position 

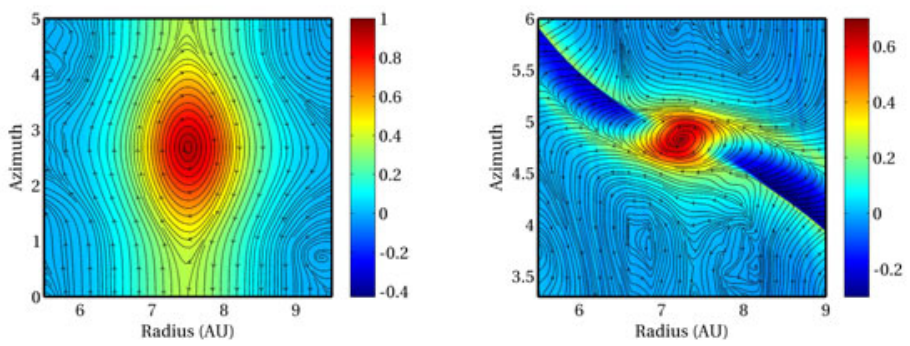

Figure 2. Density map of the two vortex families: on the left, the case of a nearly "incompressible vortex" with closed streamlines (in black), and consistently with the incompressible GNG solution; on the right, the case of a "compressible vortex" with opened streamlines and a strong spiral pattern excited by the compact core.
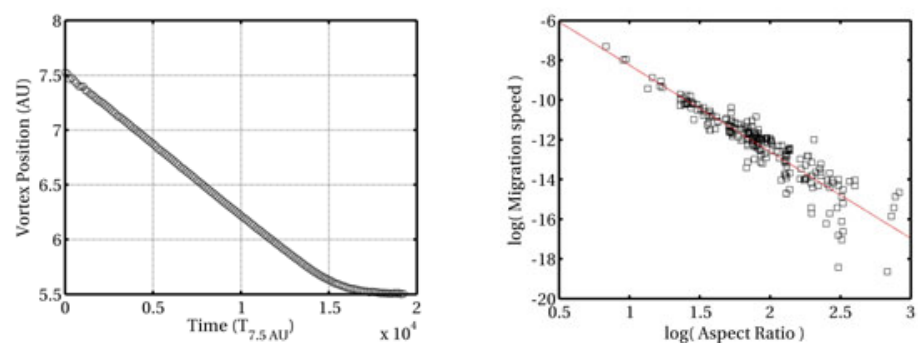

Figure 3. Vortex migration. On the left is presented the mean vortex position as a function of time (1 rotation is equivalent to 129 units on the $\mathrm{x}$-axis); the vortex reaches the inner boundary after 115 rotations and the migration speed is given by the slope of the curve. On the right is presented the correlation between the aspect ratio and the migration speed; the -4.2 slope of this curve shows the strong dependence of migration with the vortex shape.

of the vortex we determined a migration rate (figure 3) that we found independent of its radial extent. On the other hand migration is highly dependent on the aspect ratio of the vortices; the migration rate is found to scale as $\chi_{\theta}^{-4.2}$, consistent with the weak emission of spiral waves by the elongated vortices.

The migration rate of the vortices also depends on the global properties of the disk. It is varying linearly with the density and temperature gradients, as noted by [10]; we found it can also vanish if the gradients satisfy the relation $\beta_{\sigma}+\beta_{T}=3$. Finally, vortex migration is proportional to the square of the scale height, so the thicker the disk the faster the migration (fast migration is due to a fast transport of angular momentum resulting from a large sound speed in thick disks).

\section{Conclusions}

The model presented in this paper was used to construct a large number of possible vortex solutions that were tested numerically. We found that the injected vortex solutions evolve following two stages: (1) a relaxation of the initial solution toward a quasi-steady vortex, (2) a migration of the vortex toward the inner regions of the disk. The relaxation phase is found to last only a few rotation periods. During the second phase the vortex can survive a large number of rotation periods (up to 1000 rotation periods in our simulations) and migrates inward toward the star. The parameters necessary to get quasisteady vortex solutions range in a larger domain than previously thought. The migration rate is found 
to depend on the aspect ratio of the vortex and on the various parameters of the disk. Two vortex families can be distinguished following their size and shape. Large elongated vortices are weakly compressible structures that migrate very slowly toward the star. Small compact vortices are much more compressible structures that excite strong spiral waves and, thus, migrate very rapidly toward the star (migration can be as fast as $1 A U$ per 1000 years). Vortex migration can participate to the global evolution of the disk through the transport of angular momentum. This migration mechanism is due to the compression of the medium and is a robust effect. It could have a significant impact on the global disk evolution and should be included in a coherent scenario of planet formation involving vortical motions.

\section{References}

[1] Barge, P., Sommeria, J.e Astronomy E Astrophysics. 295, L1 (1995)

[2] Barranco, J. A., Marcus P.S. The Astrophysical Journal 623, 1157 (2005)

[3] Chang, P., Oishi, J.S. The Astrophysical Journal 721, 1593 (2010)

[4] Goodman, J., Narayan, R., Goldreich, P. Mon. Not. R. Astron. Soc. 225, 695 (1987)

[5] Heng, K., Kenyon, S. J. Mon. Not. R. Astron. Soc. 408, 1476 (2010)

[6] Inaba, S., Barge, P. The Astrophysical Journal 649, 415 (2006)

[7] Kida, S. Journal Phy. Soc. Japan 50, 3517 (1981)

[8] Li, H. et al. The Astrophysical Journal 533, 1023 (2000)

[9] Lovelace, R. V. E., et al. The Astrophysical Journal 513, 805 (1999)

[10] Paardekooper et al The Astrophysical Journal 725, 146 (2010) 